\title{
Formation and Biological evaluation of 4-thiazolidinone derivatives for their pharmacological activity
}

\author{
KOKILA A. PARMAR* ${ }^{*}$, and SARJU N. PARAJAPATI ${ }^{1}$ \\ Department of Chemistry, \\ H. N. G. University, Patan- 384265 \\ Gujarat, INDIA \\ ${ }^{1}$ Sheth P T Science College, Godhra \\ E-mail address: drsarjuprajapati@gmail.com
}

Keywords: 2-Aminobenzothiazole, thiazolidinone, Schiff's base, antibacterial activity.

\begin{abstract}
Thiazolidin-4-one is a versatile lead molecule for designing potential bioactive agents. An expeditious method for preparation of 4-thiazolidinones 7(a-h) are an important class of heterocycles, having potential biological importance due to their unique features. The process of convert of imine (Schiff's base) to 4-thiazolidinone through an intermediate of THF mercapto acetic acid with anhydrous zinc chloride is important synthetic method for preparation of 4-thiazolidinone. The structures of the synthesized compounds were confirmed by IR, ${ }^{1} \mathrm{H}-\mathrm{NMR},{ }^{13} \mathrm{C}$ NMR and Mass spectral studies. The compounds were screened for their antimicrobial activity against Bacillus subtilis, Staphylococcus aureus, Esherichia coli and Pseudomonas aeruginosa was determined by disc diffusion technique. All the synthesized compounds exhibited promising antimicrobial activity against the studied set of microorganisms.
\end{abstract}

\section{INTRODUCTION}

4-thiazolidinone embraces the most important position among pharmaceutically significant natural products and synthetic compounds. The role of 4-thiazolidinone nucleus in the field of medicinal chemistry provoked us to keep on working on synthesize novel derivatives having this moiety. The incredible ability of 4-thiazlidinones, to serve equally as biomimetics and reactive pharmacophore, makes up them an extremely considerable class of compounds. In view of the general observation that pharmacological activity is invariably associated with a large variety of heterocyclic compounds, the investigation of some heterocycles such as thiazolidinone derivatives has been undertaken. 4-thiazolidinones are derivatives of thiazolidine with a carbonyl group at the 4 position. Several methods for syntheses are available. The literature survey revealed that 4thiazolidinone and their derivatives were possessed a wide range of pharmacological activities such as anti-inflammatory, analgesic, anticonvulsant, antimicrobial, local and spinal anesthetics, CNS stimulants, hypnotics, anti HIV, hypoglycemic, anticancer, FSH receptor agonist and CFTR inhibitor etc [1-2]. Derivatives of these compounds are reported to possess a wide spectrum of biological activities which include anti-inflammatory, analgesic, antitumour, antihypertensive, anticonvulsant and antimicrobial activity. Owing to the importance and established physiological activity of these thiazolidinones it was thought to synthesize and investigate compounds with comparable structures [3-8]. 4-thiazolidinone nucleus has also occupied a unique place in the field of medicinal chemistry due to wide range of biological activities like antischistomal, antibacterial, anticancer, potassium channel inhibitor, cardiovascular [9], local anesthetic, sedative, cholerectic, anti-tubercular [10], antiviral, anti-tumor, CNS depressant [11], antihypertensive, hypoglycaemic[12], anti allergic [13], hypertensive, antihelmenthic, antiinflamatory, antiparkinsonian 14], hypnotic [15], antithyroidal, fungicidal[16], cysticidal, anti-leukemic [17], and anti-oxidant activities of thiadiazole, 4-thiazolidinone. The synthetic route of the abovementioned compounds is shown in reaction scheme. 


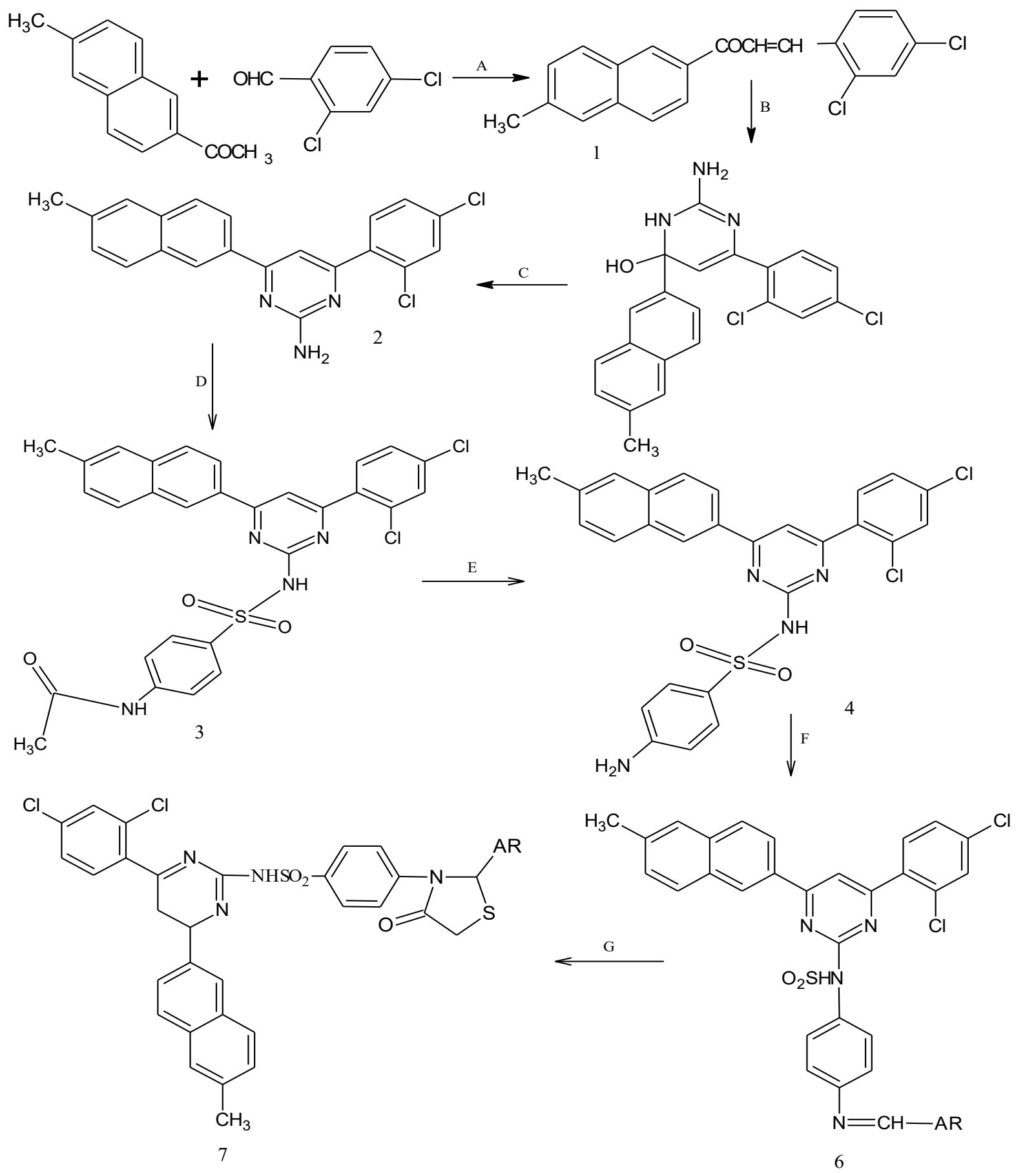

Reaction Scheme

\section{Reaction reagent and condition}

$\mathrm{A}=40 \% \mathrm{KOH}, \mathrm{OH}^{-}$

$\mathrm{E}=$ Hydrolysis

$\mathrm{B}=$ Guinidine Hydrochloride,

$\mathrm{F}=$ Different Aldehyde (5a-h)

$\mathrm{C}=-\mathrm{H}_{2} \mathrm{O},-\mathrm{H}_{2}$

$\mathrm{G}=\mathrm{THF}$ anhydrous $/ \mathrm{SNCH}_{2} \mathrm{COOH}$

$\mathrm{D}=\mathrm{P}$-Acetyl-aminobenzenesolphonyl chloride and pyridine 
Table 1: The benzaldehyde derivatives (5a-h) used for Schiff bases formation of 4-amino-N(4-(2,4-dichlorophenyl)-6-(6-methylnaphthalen-2-yl)pyrimidin-2-yl)benzenesulfonamide (4)

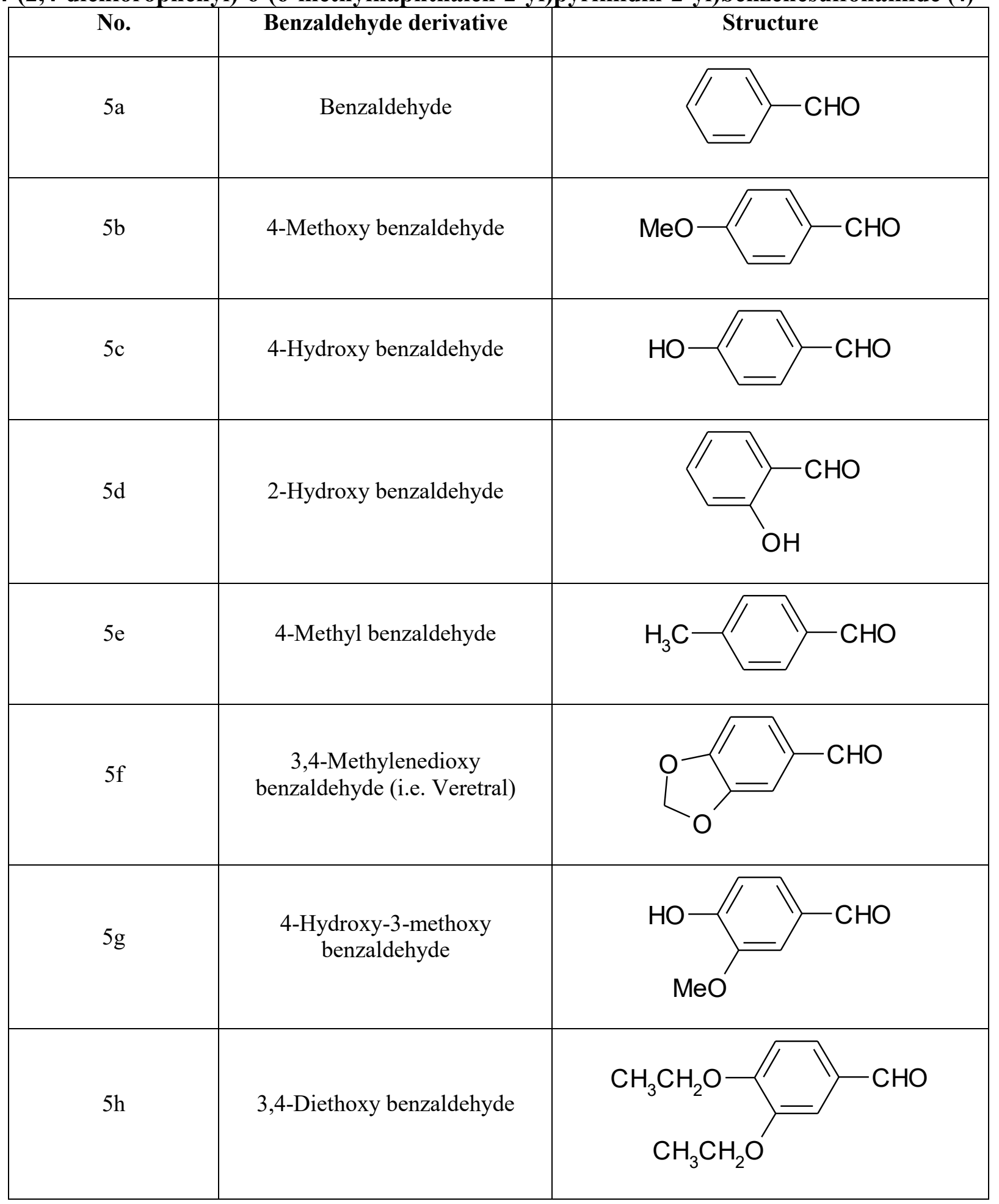


Table 2: Physical characterisation constant of 4-(benzylideneamino)-N-(4-(2,4dichlorophenyl)-6-(6-methylnaphthalen-2-yl)pyrimidin-2-yl)benzenesulfonamide (6 a-h)

\begin{tabular}{|c|c|c|c|c|c|c|c|}
\hline \multirow{2}{*}{$\begin{array}{c}\text { Com. } \\
\text { No }\end{array}$} & \multirow{2}{*}{$\begin{array}{l}\text { Molecular } \\
\text { Formula }\end{array}$} & \multirow{2}{*}{$-\mathrm{Ar}$} & \multirow{2}{*}{$\begin{array}{c}\text { Molecular } \\
\text { Weight }\end{array}$} & \multicolumn{4}{|c|}{ Elemental analysis, Cal/Found } \\
\hline & & & & $\% \mathrm{C}$ & $\% \mathrm{H}$ & $\% \mathrm{~N}$ & $\% \mathrm{~S}$ \\
\hline \multirow[t]{2}{*}{$6 a$} & $\mathrm{C}_{34} \mathrm{H}_{24} \mathrm{Cl}_{2} \mathrm{~N}_{4} \mathrm{O}_{2} \mathrm{~S}$ & Phenyl & 622.10 & 65.49 & 3.88 & 8.99 & 5.14 \\
\hline & & & gm $/$ mole & 65.19 & 3.57 & 8.89 & 5.10 \\
\hline \multirow[t]{2}{*}{$6 b$} & $\mathrm{C}_{35} \mathrm{H}_{26} \mathrm{Cl}_{2} \mathrm{~N}_{4} \mathrm{O}_{3} \mathrm{~S}$ & 4-Methoxy phenyl & 652.11 & 64.32 & 4.01 & 8.57 & 4.91 \\
\hline & & & $\mathrm{gm} /$ mole & 64.12 & 3.92 & 8.51 & 4.86 \\
\hline \multirow[t]{2}{*}{$6 c$} & $\mathrm{C}_{34} \mathrm{H}_{24} \mathrm{Cl}_{2} \mathrm{~N}_{4} \mathrm{O}_{3} \mathrm{~S}$ & 4-Hydroxy phenyl & 638.09 & 63.85 & 3.78 & 8.76 & 5.01 \\
\hline & & & $\mathrm{gm} / \mathrm{mole}$ & 63.55 & 3.65 & 8.56 & 5.00 \\
\hline \multirow[t]{2}{*}{$6 \mathrm{~d}$} & $\mathrm{C}_{34} \mathrm{H}_{24} \mathrm{Cl}_{2} \mathrm{~N}_{4} \mathrm{O}_{3} \mathrm{~S}$ & 2-Hydroxy phenyl & 638.09 & 63.85 & 3.78 & 8.76 & 5.01 \\
\hline & & & $\mathrm{gm} / \mathrm{mole}$ & 63.55 & 3.65 & 8.56 & 5.00 \\
\hline \multirow[t]{2}{*}{$6 e$} & $\mathrm{C}_{34} \mathrm{H}_{26} \mathrm{Cl}_{2} \mathrm{~N}_{4} \mathrm{O}_{2} \mathrm{~S}$ & 4-Methyl phenyl & 636.12 & 63.93 & 4.11 & 8.89 & 5.03 \\
\hline & & & gm $/$ mole & 63.73 & 4.01 & 8.79 & 5.00 \\
\hline \multirow[t]{2}{*}{$6 f$} & $\mathrm{C}_{35} \mathrm{H}_{24} \mathrm{Cl}_{2} \mathrm{~N}_{4} \mathrm{O}_{4} \mathrm{~S}$ & 3,4-Methylenedioxy & 666.09 & 62.97 & 3.62 & 8.39 & 4.80 \\
\hline & & phenyl & $\mathrm{gm} / \mathrm{mole}$ & 62.81 & 3.55 & 8.32 & 4.70 \\
\hline \multirow[t]{2}{*}{$6 g$} & $\mathrm{C}_{35} \mathrm{H}_{26} \mathrm{Cl}_{2} \mathrm{~N}_{4} \mathrm{O}_{4} \mathrm{~S}$ & 4-Hydroxy-3- & 668.11 & 62.78 & 3.91 & 8.37 & 4.79 \\
\hline & & methoxy phenyl & $\mathrm{gm} / \mathrm{mole}$ & 62.68 & 3.76 & 8.30 & 4.73 \\
\hline \multirow[t]{2}{*}{$6 \mathrm{~h}$} & $\mathrm{C}_{38} \mathrm{H}_{32} \mathrm{Cl}_{2} \mathrm{~N}_{4} \mathrm{O}_{4} \mathrm{~S}$ & 3,4-Diethoxy phenyl & 710.19 & 64.13 & 4.53 & 7.87 & 4.51 \\
\hline & & & gm $/$ mole & 64.00 & 4.48 & 7.81 & 4.44 \\
\hline
\end{tabular}

Table 3: Physical characterisation constant of N-(4-(2,4- dichlorophenyl)-6-(6methylnaphthalen-2-yl)pyrimidin-2-yl)-4-(4-oxo-2-phenylthiazolidin-3-yl)benzenesulfonamide (7a-h)

\begin{tabular}{|c|c|c|c|c|c|c|c|}
\hline \multirow{2}{*}{$\begin{array}{l}\text { Com } \\
\text {. No }\end{array}$} & \multirow{2}{*}{$\begin{array}{l}\text { Molecular } \\
\text { Formula }\end{array}$} & \multirow{2}{*}{$-\mathrm{Ar}$} & \multirow{2}{*}{$\begin{array}{l}\text { Molecular } \\
\text { Weight }\end{array}$} & \multicolumn{4}{|c|}{ Elemental analysis, Cal/Found } \\
\hline & & & & $\% \mathrm{C}$ & $\% \mathrm{H}$ & $\% \mathrm{~N}$ & $\% \mathrm{~S}$ \\
\hline \multirow[t]{2}{*}{$7 \mathrm{a}$} & $\mathrm{C}_{36} \mathrm{H}_{26} \mathrm{Cl}_{2} \mathrm{~N}_{4} \mathrm{O}_{3} \mathrm{~S}_{2}$ & Methylbenzene & & 61.98 & 3.76 & 8.03 & 9.19 \\
\hline & & & $\mathrm{gm} / \mathrm{mole}$ & 61.80 & 3.71 & 8.00 & 9.01 \\
\hline \multirow[t]{2}{*}{$7 b$} & $\mathrm{C}_{37} \mathrm{H}_{28} \mathrm{Cl}_{2} \mathrm{~N}_{4} \mathrm{O}_{4} \mathrm{~S}_{2}$ & 1-Methoxy-4- & 726.09 & 61.07 & 3.88 & 7.70 & 8.81 \\
\hline & & methylbenzene & $\mathrm{gm} / \mathrm{mole}$ & 61.00 & 3.75 & 7.62 & 8.60 \\
\hline \multirow[t]{2}{*}{$7 \mathrm{c}$} & $\mathrm{C}_{36} \mathrm{H}_{26} \mathrm{Cl}_{2} \mathrm{~N}_{4} \mathrm{O}_{4} \mathrm{~S}_{2}$ & 4-Methylphenol & 712.19 & 60.59 & 3.67 & 7.85 & 8.99 \\
\hline & & & $\mathrm{gm} / \mathrm{mole}$ & 60.46 & 3.60 & 7.80 & 8.78 \\
\hline \multirow[t]{2}{*}{$7 d$} & $\mathrm{C}_{36} \mathrm{H}_{26} \mathrm{Cl}_{2} \mathrm{~N}_{4} \mathrm{O}_{4} \mathrm{~S}_{2}$ & 2-Methylphenol & 712.19 & 60.59 & 3.67 & 7.85 & 8.99 \\
\hline & & & $\mathrm{gm} / \mathrm{mole}$ & 60.48 & 3.55 & 7.77 & 8.79 \\
\hline \multirow[t]{2}{*}{$7 \mathrm{e}$} & $\mathrm{C}_{37} \mathrm{H}_{28} \mathrm{Cl}_{2} \mathrm{~N}_{4} \mathrm{O}_{3} \mathrm{~S}_{2}$ & 1,4 & 710.10 & 62.44 & 3.97 & 7.87 & 9.01 \\
\hline & & Dimethylbenzene & $\mathrm{gm} / \mathrm{mole}$ & 62.40 & 3.93 & 7.83 & 8.89 \\
\hline \multirow[t]{2}{*}{$7 f$} & $\mathrm{C}_{37} \mathrm{H}_{26} \mathrm{Cl}_{2} \mathrm{~N}_{4} \mathrm{O}_{5} \mathrm{~S}_{2}$ & 5-Methyl-1,3- & 740.17 & 59.92 & 3.53 & 7.55 & 8.65 \\
\hline & & benzodioxole & $\mathrm{gm} / \mathrm{mole}$ & 59.89 & 3.50 & 7.50 & 8.61 \\
\hline \multirow[t]{2}{*}{$7 \mathrm{~g}$} & $\mathrm{C}_{37} \mathrm{H}_{28} \mathrm{Cl}_{2} \mathrm{~N}_{4} \mathrm{O}_{5} \mathrm{~S}_{2}$ & 2-Methoxy-4- & 742.09 & 59.76 & 3.79 & 7.53 & 8.62 \\
\hline & & methylphenol & $\mathrm{gm} / \mathrm{mole}$ & 59.70 & 3.73 & 7.48 & 8.60 \\
\hline \multirow[t]{2}{*}{$7 \mathrm{~h}$} & $\mathrm{C}_{40} \mathrm{H}_{34} \mathrm{Cl}_{2} \mathrm{~N}_{4} \mathrm{O}_{5} \mathrm{~S}_{2}$ & 1,2- Diethoxy-4- & 784.13 & 61.14 & 4.36 & 7.13 & 8.16 \\
\hline & & methylbenzene & $\mathrm{gm} / \mathrm{mole}$ & 61.03 & 4.31 & 7.10 & 8.03 \\
\hline
\end{tabular}




\section{METHODOLOGY}

Synthesis of 3-(2,4-dichlorophenyl)-1-(6-methylnaphthalen-2-yl)prop-2-en-1-one (1)

To a well stirred solution of 2,4-dichloro benzaldehyde (0.01 mole) and 1-(6-methylnaphthalen-2yl)ethanone $(0.01 \mathrm{~mole})$ in ethanol $(35 \mathrm{ml}), 40 \% \mathrm{KOH}$ added till the solution become basic. The reaction mixture was stirred for $24 \mathrm{hrs}$. The contents were poured into ice, acidified, filtered and crystallized from ethanol.

Synthesis of 4-amino-N-(4-(2,4-dichlorophenyl)-6-(6-methylnaphthalen-2-yl)pyrimidin-2yl)benzenesulfonamide (2)

A mixture of Chalcone ( 0.01 mole) in $25 \mathrm{ml}$ of absolute alcohol, add Guanidine Hydrochloride ( 0.015 mole) and sodium hydroxide ( 0.045 mole in $2 \mathrm{ml}$ of water) was refluxed in water bath at temp $80-90^{\circ} \mathrm{c}$ for $8 \mathrm{hr}$. The reaction mixture was poured into ice. The product was isolated and crystallized from ethanol.

Synthesis of N-(4-(N-(4-(2,4-dichlorophenyl)-6-(6-methylnaphthalen-2-yl)pyrimidin-2yl)sulfamoyl)phenyl)acetamide (3)

The derivative was prepared by reactions of amino pyrimidine (2) $(0.01$ mole $)$ with $P$-Acetylaminobenzenesulfonyl chloride $(0.012 \mathrm{~mole})$ in dry pyridine $(30 \mathrm{ml})$ was heated to $70-75^{\circ} \mathrm{C}$ on a water bath for $5 \mathrm{hr}$. the cold reaction mixture was acidified with dil. hydrochloric acid. The solid that separated was filtered, washed several times with hot water, dried and crystallized from proper solvent.

\section{4-amino-N-(4-(2,4-dichlorophenyl)-6-(6-methylnaphthalen-2-yl)pyrimidin-2-} yl)benzenesulfonamide (4)

N-(4-(N-(4-(2,4-dichlorophenyl)-6-(6-methylnaphthalen-2-yl)pyrimidin-2-

yl)sulfamoyl)phenyl)acetamide (3) was hydrolysed by refluxing 0.5-1.0 molar solution containing 3.5 equivalents of sodium hydroxide for two hours. After this period, the mixture was cooled to room temperature and neutralized with concentrated $\mathrm{HCl} \mathrm{pH}$ by approximately 6.0. The mixture was cooled in the ice bath until the total precipitation of the product, the filtered vacuum, washed with small volume of water ice and purification by recrystallization from ethanol to give white product.

Synthesis of 4-(benzylideneamino)-N-(4-(2,4-dichlorophenyl)-6-(6-methylnaphthalen-2yl)pyrimidin-2-yl)benzenesulfonamide (6 a-h)

The various Schiff bases (6a-h) of 4-amino-N-(4-(2,4-dichlorophenyl)-6-(6-methylnaphthalen-2yl)pyrimidin-2-yl)benzenesulfonamide (4) have been prepared in the similar manner. The procedure is as follow:

A mixture of equimolar amount (0.01 mole) of of 4-amino-N-(4-(2,4-dichlorophenyl)-6-(6methylnaphthalen-2-yl)pyrimidin-2-yl)benzenesulfonamide (4) and the Substituted Benzaldehydes (5) in absolute ethanol $(70 \mathrm{ml})$ and piperidine $(0.5 \mathrm{ml})$ was refluxed for $10 \mathrm{hr}$. in a water bath. The reaction mixture was concentrated, cooled and poured into ice cold water the solid obtained was filtered and Recrystallized from absolute ethanol to give white Schiff base.

Synthesis of N-(4-(2,4-dichlorophenyl)-6-(6-methylnaphthalen-2-yl)pyrimidin-2-yl)-4-(4-oxo2-phenylthiazolidin-3-yl)benzenesulfonamide (7a-h)

A mixture of Schiff bases $(\mathbf{6 a - h})(0.01 \mathrm{~mole})$ in THF $(30 \mathrm{ml})$ and mercapto acetic acid (thioglycolic acid) $\left(0.01\right.$ mole) with a pinch of anhydrous $\mathrm{ZnCl}_{2}$ was refluxed for 12 hours. The solvent was then removed to get a residue, which was dissolved in benzene and passed through column of silica gel using benzene: chloroform $(8: 2 ; \mathrm{v} / \mathrm{v})$ mixture as eluent. The eluate was concentrated and the product crystallized from alcohol to give 4-thiazolidinones (7a-h), which were obtained in 50-60\% yield. 


\section{RESULTS AND DISCUSSION \\ SPECTRAL STUDIES:}

IR spectra of 4-amino-N-(4-(2,4-dichlorophenyl)-6-(6-methylnaphthalen-2-yl)pyrimidin-2yl)benzenesulfonamide (4)

Sulfa Pyrimidine is a heterocyclic compound. It is an aromatic compound thus it provides the IR frequencies. The bands due to pyrimidine are at $3220-3440 \mathrm{~cm}^{-1}$ and $1610-1640 \mathrm{~cm}^{-1}$ corresponds to $\mathrm{N}-\mathrm{H}$ (str.) and $\mathrm{C}=\mathrm{N}$ groups. The peak at $1630 \mathrm{~cm}^{-1}$ is indicative of $\mathrm{C}=\mathrm{N}, 1600 \mathrm{~cm}^{-1}$ due to $\mathrm{C}=\mathrm{C} \mathrm{cm}^{-1}$, and $1310 \mathrm{~cm}^{-1}$ and $1150 \mathrm{~cm}^{-1}$ diagnostic for the presence of the sulphonamido group(-SO2NH-) also The corresponding N-H in plane and out of plane bending vibrations occurs at 1630 and $699 \mathrm{~cm}^{-1}$ respectively.

NMR spectra of 4-amino-N-(4-(2,4-dichlorophenyl)-6-(6-methylnaphthalen-2-yl)pyrimidin-2yl)benzenesulfonamide (4)

The signal at $4.0 \mathrm{ppm}$ is responsible for $\mathrm{N}-\mathrm{H}$ proton of pyrimidine $-\mathrm{SO}_{2} \mathrm{NH}-$, signal at $6.35 \mathrm{ppm}$ is responsible for $-\mathrm{NH}_{2}$ proton, and multiple signals between 6.15-7.8 ppm are responsible for aromatic proton. While signal at 2.30 due to two $-\mathrm{CH}_{3}$ on benzene ring.

CMR spectra of 4-amino-N-(4-(2,4-dichlorophenyl)-6-(6-methylnaphthalen-2-yl)pyrimidin-2yl)benzenesulfonamide (4)

The signals at $101.7\left(\mathrm{C}_{5}\right), 162.5\left(\mathrm{C}_{4}\right), 165.4\left(\mathrm{C}_{6}\right)$ and $169.3\left(\mathrm{C}_{2}\right)$ ppm are responsible for pyrimidine multiple signals between 114-140 ppm are responsible for aromatic segments. While signal at 18.8 and 19.1 are due to two $-\mathrm{CH}_{3}$.

Finally the structure of compound conform by LC-MS compound (4) shows peak of (m/Z) at 534.07 which consistent with the calculated molecular weight of Compound (4) i.e. 534.07.

Spectral Studies of compound 6a-6h

4-(benzylideneamino)-N-(4-(2,4-dichlorophenyl)-6-(6-methylnaphthalen-2-yl)pyrimidin-2yl)benzenesulfonamide $6 \mathrm{a}$

Yield: $60 \%$, MP $193^{0} \mathrm{C}$, Infrared Spectral Features around $\mathrm{cm}^{-1} 3030$, 1500, Aromatic C-H stretching, 1600- $1641-\mathrm{CH}=\mathrm{N}-$, 1315-1375 - $\mathrm{SO}_{2-}, 3250-3330-\mathrm{NH}-$ of $-\mathrm{SO}_{2} \mathrm{NH}-$, PMR spectral Features $(\delta \mathrm{Ppm})$ 6.5-8.5 multiplet, aromatic $+\mathrm{CH}$ of $\mathrm{CH}=\mathrm{N}$ protons $+\mathrm{H}$ of Pyrimdine $+\mathrm{H}$ of $\left.\mathrm{SO}_{2} \mathrm{NH}+\right),{ }^{13} \mathrm{CMR}$ spectral Features $(\delta \mathrm{Ppm})$ 114-131- Benzene, 134- Ar-Cl, 160- $\mathrm{CH}=\mathrm{N}, 162-169$ pyrimidine.

N-(4-(2,4-dichlorophenyl)-6-(6-methylnaphthalen-2-yl)pyrimidin-2-yl)-4-(4methoxybenzylideneamino)benzenesulfonamide $6 \mathrm{~b}$

Yield: $61 \%$, MP $197^{0} \mathrm{C}$, Infrared Spectral Features around $\mathrm{cm}^{-1} 3030$, 1500, Aromatic C-H stretching, 1600-1640 - $\mathrm{CH}=\mathrm{N}-, 1315-1375-\mathrm{SO}_{2-}, 3250-3330-\mathrm{NH}-$ of - $\mathrm{SO}_{2} \mathrm{NH}-$, 1200- Ar-O-alkyl, PMR spectral Features $(\delta \mathrm{Ppm})$ 6.5-8.6 (multiplet, aromatic $+\mathrm{CH}$ of $\mathrm{CH}=\mathrm{N}$ protons $+\mathrm{H}$ of Pyrimdine $+\mathrm{H}$ of $\left.\mathrm{SO}_{2} \mathrm{NH}\right) 3.85\left(3 \mathrm{H}\right.$, singlet, $\left.\mathrm{OCH}_{3}\right),{ }^{13} \mathrm{CMR}$ spectral Features $(\delta \mathrm{Ppm}) 114-131$ Benzene, 134- $\mathrm{Ar}-\mathrm{Cl}, 160-\mathrm{CH}=\mathrm{N}, 162-169$ - pyrimidine, 163-C-O, $56-\mathrm{CH}_{3}$

N-(4-(2,4-dichlorophenyl)-6-(6-methylnaphthalen-2-yl)pyrimidin-2-yl)-4-(4hydroxybenzylideneamino)benzenesulfonamide $6 \mathrm{c}$

Yield: $60 \%$, MP $199^{0} \mathrm{C}$, Infrared Spectral Features around $\mathrm{cm}^{-1} 3370-\mathrm{OH}, 3030,1500$, Aromatic C-

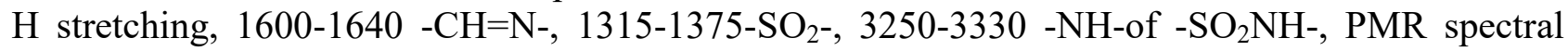
Features $(\delta \mathrm{Ppm})$ 6.5-8.6 (multiplet, aromatic $+\mathrm{CH}$ of $\mathrm{CH}=\mathrm{N}$ protons $+\mathrm{H}$ of Pyrimdine $+\mathrm{H}$ of $\left.\mathrm{SO}_{2} \mathrm{NH}\right) 3.85\left(3 \mathrm{H}\right.$, singlet, $\left.\mathrm{OCH}_{3}\right),{ }^{13} \mathrm{CMR}$ spectral Features $(\delta \mathrm{Ppm}) 114-131$-Benzene, $134-\mathrm{Ar}-\mathrm{Cl}$, $160-\mathrm{CH}=\mathrm{N}, 162-169$ - pyrimidine, $163-\mathrm{C}-\mathrm{O}$.

N-(4-(2,4-dichlorophenyl)-6-(6-methylnaphthalen-2-yl)pyrimidin-2-yl)-4-(2-

hydroxybenzylideneamino)benzenesulfonamide $6 \mathrm{~d}$

Yield: $62 \%$, MP $191{ }^{0} \mathrm{C}$, Infrared Spectral Features around $\mathrm{cm}^{-1} 3370-\mathrm{OH}, 3030,1500$, Aromatic C$\mathrm{H}$ stretching, 1600-1640 - $\mathrm{CH}=\mathrm{N}-$, , 1315-1375- $\mathrm{SO}_{2-}$, 3250-3330-NH- of $-\mathrm{SO}_{2} \mathrm{NH}-, \mathrm{PMR}$ spectral Features $(\delta \mathrm{Ppm}) 6.5-8.6$ (multiplet, aromatic $+\mathrm{CH}$ of $\mathrm{CH}=\mathrm{N}$ protons $+\mathrm{H}$ of Pyrimdine $+\mathrm{H}$ of $\left.\mathrm{SO}_{2} \mathrm{NH}\right) 3.85\left(3 \mathrm{H}\right.$, singlet, $\left.\mathrm{OCH}_{3}\right),{ }^{13} \mathrm{CMR}$ spectral Features $(\delta \mathrm{Ppm}) 114-131-B e n z e n e, 134-\mathrm{Ar}-\mathrm{Cl}$, $160-\mathrm{CH}=\mathrm{N}, 162-169$ - pyrimidine, $163-\mathrm{C}-\mathrm{O}$. 
N-(4-(2,4-dichlorophenyl)-6-(6-methylnaphthalen-2-yl)pyrimidin-2-yl)-4-(4methylbenzylideneamino)benzenesulfonamide $6 \mathrm{e}$

Yield: $62 \%$, MP $193^{\circ} \mathrm{C}$, Infrared Spectral Features around $\mathrm{cm}^{-1} 2950,1370-\mathrm{CH}_{3}, 3030,1500$, Aromatic C-H stretching, 1600-1640 - $\mathrm{CH}=\mathrm{N}-$, , 1315-1375 - $\mathrm{SO}_{2^{-}}, 3250-3330-\mathrm{NH}-$ of $-\mathrm{SO}_{2} \mathrm{NH}-$, PMR spectral Features $(\delta \mathrm{Ppm})$ 6.5-8.7 (multiplet, aromatic $+\mathrm{CH}$ of $\mathrm{CH}=\mathrm{N}$ protons $+\mathrm{H}$ of Pyrimdine $+\mathrm{H}$ of $\left.\mathrm{SO}_{2} \mathrm{NH}\right) 2.34\left(3 \mathrm{H}\right.$, singlet, $\left.\mathrm{CH}_{3}\right),{ }^{13} \mathrm{CMR}$ spectral Features $(\delta \mathrm{Ppm}) 114-131-$ Benzene, 134- Ar-Cl, 160 - $\mathrm{CH}=\mathrm{N}, 162-169$ - pyrimidine, 21- $\mathrm{CH}_{3}$.

4-(benzo[d][1,3]dioxol-5-ylmethyleneamino)-N-(4-(2,4-dichlorophenyl)-6-(6-

methylnaphthalen-2-yl)pyrimidin-2-yl)benzenesulfonamide $6 f$

Yield: $60 \%$, MP $201^{\circ} \mathrm{C}$, Infrared Spectral Features around $\mathrm{cm}^{-1} 2920,2850-\mathrm{CH}_{2-}, 1450,3030$, 1500, Aromatic C-H stretching, 1600-1640 - $\mathrm{CH}=\mathrm{N}-$, , 1315-1365 - $\mathrm{SO}_{2-}$, 3250-3330 -NH- of $\mathrm{SO}_{2} \mathrm{NH}-$, PMR spectral Features $(\delta \mathrm{Ppm})$ 6.5-8.7 (multiplet, aromatic $+\mathrm{CH}$ of $\mathrm{CH}=\mathrm{N}$ protons $+\mathrm{H}$ of Pyrimdine $+\mathrm{H}$ of $\left.\mathrm{SO}_{2} \mathrm{NH}\right) 6.07\left(2 \mathrm{H}\right.$, singlet, $\left.-\mathrm{O}-\mathrm{CH}_{2}-\mathrm{O}-\right),{ }^{13} \mathrm{CMR}$ spectral Features $(\delta \mathrm{Ppm})$ 101.2 - $\mathrm{CH}_{2}-\mathrm{O}-$, , 114-131-Benzene, 134- $\mathrm{Ar}-\mathrm{Cl}, 160-\mathrm{CH}=\mathrm{N}, 162-169$ - pyrimidine, $21-\mathrm{CH}_{3}$.

N-(4-(2,4-dichlorophenyl)-6-(6-methylnaphthalen-2-yl)pyrimidin-2-yl)-4-(3-hydroxy-4methoxybenzylideneamino)benzenesulfonamide $6 \mathrm{~g}$

Yield: $57 \%$, MP $199^{0} \mathrm{C}$, Infrared Spectral Features around $\mathrm{cm}^{-1} 3370-\mathrm{OH}, 2950,1370-\mathrm{CH}_{3}$, 3030,1500, Aromatic C-H stretching, 1600-1640 -CH=N-, 1315-1375 - $\mathrm{SO}_{2-}$, 3250-3330 -NH- of $\mathrm{SO}_{2} \mathrm{NH}-$, PMR spectral Features $(\delta \mathrm{Ppm})$ 6.5-8.7 (multiplet, aromatic $+\mathrm{CH}$ of $\mathrm{CH}=\mathrm{N}$ protons $+\mathrm{H}$ of Pyrimdine $+\mathrm{H}$ of $\left.\mathrm{SO}_{2} \mathrm{NH}\right) 3.83\left(3 \mathrm{H}\right.$, singlet, $\left.-\mathrm{O}-\mathrm{CH}_{3}\right) 5.35(1 \mathrm{H}$, Singlet, $-\mathrm{OH}),{ }^{13} \mathrm{CMR}$ spectral Features $(\delta \mathrm{Ppm})$ 114-131-Benzene, 134- $\mathrm{Ar}-\mathrm{Cl}, 160-\mathrm{CH}=\mathrm{N}, 162-169$-pyrimidine, 149-151 -C $\mathrm{O}, 56-\mathrm{OCH}_{3}$.

N-(4-(2,4-dichlorophenyl)-6-(6-methylnaphthalen-2-yl)pyrimidin-2-yl)-4-(3,4-

diethoxybenzylideneamino)benzenesulfonamide $6 \mathrm{~h}$

Yield: $60 \%$, MP $190^{\circ} \mathrm{C}$, Infrared Spectral Features around $\mathrm{cm}^{-1} 2950,2820,-\mathrm{CH}_{2-}, 1450,3030,1500$, Aromatic C-H stretching, 1600-1640 - $\mathrm{CH}=\mathrm{N}-$, , 1315-1375 - $\mathrm{SO}_{2-}$, 3250-3330 -NH- of - $\mathrm{SO}_{2} \mathrm{NH}-$, PMR spectral Features $(\delta \mathrm{Ppm})$ 6.5-8.7 (multiplet, aromatic $+\mathrm{CH}$ of $\mathrm{CH}=\mathrm{N}$ protons $+\mathrm{H}$ of Pyrimdine $+\mathrm{H}$ of $\left.\mathrm{SO}_{2} \mathrm{NH}\right) 4.0\left(4 \mathrm{H}\right.$, quartet, $\left.2 \mathrm{CH}_{2}\right) 1.33\left(6 \mathrm{H}\right.$, triplet, $\left.2 \mathrm{CH}_{3}\right),{ }^{13} \mathrm{CMR}$ spectral Features $(\delta \mathrm{Ppm})$ 114-131-Benzene, 134- Ar-Cl, $160-\mathrm{CH}=\mathrm{N}, 162-169$ - pyrimidine, 149-151 -C-O.

N-(4-(2,4-dichlorophenyl)-6-(6-methylnaphthalen-2-yl)pyrimidin-2-yl)-4-(4-oxo-2-

phenylthiazolidin-3-yl)benzenesulfonamide $7 \mathbf{a}$

Yield: $64 \%$, MP $198-99^{0} \mathrm{C}$, Infrared Spectral Features around $\mathrm{cm}^{-1} 3030$, 1500, Aromatic C-H stretching, $1600-1641-\mathrm{CH}=\mathrm{N}, 1315-1375-\mathrm{SO}_{2^{-}}, 3250-3330-\mathrm{NH}-$ of $-\mathrm{SO}_{2} \mathrm{NH}-, 1690 \mathrm{C}=\mathrm{O}$ of thiazolidinone, PMR spectral Features $(\delta \mathrm{Ppm}) 6.5-7.9$ (multiplet, aromatic $+\mathrm{H}$ of Pyrimdine), 3.9-4.0 (2H of $\mathrm{CH}_{2}$ for thiazolidinone), $6.44\left(\mathrm{H}\right.$ of $\mathrm{C}_{2} \mathrm{H}$ for thiazolidinone), $11.34\left(\mathrm{H}\right.$ of $\left.\mathrm{SO}_{2} \mathrm{NH}\right)$, ${ }^{13} \mathrm{CMR}$ spectral Features $(\delta \mathrm{Ppm}) 114-131$ - Benzene, 134- $\mathrm{Ar}-\mathrm{Cl}, 163-169$ - pyrimidine, $171.2 \mathrm{C}=\mathrm{O}$, $33.5 \mathrm{CH}_{2}, 65.6 \mathrm{CH}$.

N-(4-(2,4-dichlorophenyl)-6-(6-methylnaphthalen-2-yl)pyrimidin-2-yl)-4-(2-(4methoxyphenyl)-4-oxothiazolidin-3-yl)benzenesulfonamide $7 \mathrm{~b}$

Yield: $60 \%$, MP $191-92^{0} \mathrm{C}$, Infrared Spectral Features around $\mathrm{cm}^{-1} 3030$, 1500, Aromatic C-H stretching, 1600-1641 $-\mathrm{CH}=\mathrm{N}, 1315-1375-\mathrm{SO}_{2^{-}}, 3250-3330-\mathrm{NH}-$ of $-\mathrm{SO}_{2} \mathrm{NH}-, 1690 \mathrm{C}=\mathrm{O}$ of thiazolidinone, 1200 Aryl-alkyl ether, PMR spectral Features $(\delta \mathrm{Ppm})$ 6.5-7.9 (multiplet, aromatic $+\mathrm{H}$ of Pyrimdine $+\mathrm{H}$ of $\left.\mathrm{SO}_{2} \mathrm{NH}\right), 6.44\left(\mathrm{H}\right.$ of $\mathrm{C}_{2} \mathrm{H}$ for thiazolidinone), $3.83\left(3 \mathrm{H}\right.$, singlet, $\left.\mathrm{OCH}_{3}\right)$ $11.34\left(\mathrm{H}\right.$ of $\left.\mathrm{SO}_{2} \mathrm{NH}\right),{ }^{13} \mathrm{CMR}$ spectral Features $(\delta \mathrm{Ppm})$ 114-131- Benzene, 163-169- pyrimidine, $171.2 \mathrm{C}=\mathrm{O}, 33.5 \mathrm{CH}_{2}, 65.6 \mathrm{CH}, 158-159-\mathrm{C}-\mathrm{O}, 56 \mathrm{CH}_{3}$.

N-(4-(2,4-dichlorophenyl)-6-(6-methylnaphthalen-2-yl)pyrimidin-2-yl)-4-(2-(4hydroxyphenyl)-4-oxothiazolidin-3-yl)benzenesulfonamide 7c

Yield: $55 \%$, MP $189-90^{0} \mathrm{C}$, Infrared Spectral Features around $\mathrm{cm}^{-1} 3030$, 1500, Aromatic C-H stretching, 1600-1641 $-\mathrm{CH}=\mathrm{N}, 1315-1375-\mathrm{SO}_{2-}, 3250-3330-\mathrm{NH}-$ of $-\mathrm{SO}_{2} \mathrm{NH}-, 1690 \mathrm{C}=\mathrm{O}$ of thiazolidinone, 3200,2600 -OH Phenolic (broad), 2880,2920,1400 $\mathrm{CH}_{2}, 1200$ Aryl-alkyl ether, PMR spectral Features $(\delta \mathrm{Ppm})$ 6.5-7.9 (multiplet, aromatic $+\mathrm{H}$ of Pyrimdine $+\mathrm{H}$ of $\left.\mathrm{SO}_{2} \mathrm{NH}\right), 6.44(\mathrm{H}$ 
of $\mathrm{C}_{2} \mathrm{H}$ for thiazolidinone), $11.34\left(\mathrm{H}\right.$ of $\left.\mathrm{SO}_{2} \mathrm{NH}\right), 5.35(\mathrm{H}$ of OH$),{ }^{13} \mathrm{CMR}$ spectral Features $(\delta \mathrm{Ppm})$ 114-131- Benzene, $134 \mathrm{Ar}-\mathrm{Cl}, 163-169$ - pyrimidine, 171.2- $\mathrm{C}=\mathrm{O}, 33.5 \mathrm{CH}_{2}, 65.6 \mathrm{CH}$.

N-(4-(2,4-dichlorophenyl)-6-(6-methylnaphthalen-2-yl)pyrimidin-2-yl)-4-(2-(2hydroxyphenyl)-4-oxothiazolidin-3-yl)benzenesulfonamide 7d

Yield: $59 \%$, MP $186-86^{\circ} \mathrm{C}$, Infrared Spectral Features around $\mathrm{cm}^{-1} 3030$, 1500, Aromatic C-H stretching, 1600-1641 $-\mathrm{CH}=\mathrm{N}, 1315-1375-\mathrm{SO}_{2-}, 3250-3330-\mathrm{NH}-$ of $-\mathrm{SO}_{2} \mathrm{NH}-, 1690 \mathrm{C}=\mathrm{O}$ of thiazolidinone, 3200,2600 -OH Phenolic (broad), 2880,2920,1400 $\mathrm{CH}_{2}, \mathrm{PMR}$ spectral Features $(\delta$ Ppm) 6.5-7.9 (multiplet, aromatic $+\mathrm{H}$ of Pyrimdine $+\mathrm{H}$ of $\left.\mathrm{SO}_{2} \mathrm{NH}\right), 6.44\left(\mathrm{H}\right.$ of $\mathrm{C}_{2} \mathrm{H}$ for thiazolidinone), 3.9-4.0 (2H of $\mathrm{CH}_{2}$ for thiazolidinone), $11.34\left(\mathrm{H}\right.$ of $\left.\mathrm{SO}_{2} \mathrm{NH}\right), 5.35(\mathrm{H}$ of $\mathrm{OH})$, ${ }^{13} \mathrm{CMR}$ spectral Features $(\delta \mathrm{Ppm}) 114-131$ - Benzene, $134 \mathrm{Ar}-\mathrm{Cl}, 163-169$ - pyrimidine, $171.2-\mathrm{C}=\mathrm{O}$, $33.5 \mathrm{CH}_{2}, 65.6 \mathrm{CH}$.

N-(4-(2,4-dichlorophenyl)-6-(6-methylnaphthalen-2-yl)pyrimidin-2-yl)-4-(4-oxo-2-ptolylthiazolidin-3-yl)benzenesulfonamide $7 \mathrm{e}$

Yield: $60 \%$, MP $195-96^{0} \mathrm{C}$, Infrared Spectral Features around $\mathrm{cm}^{-1} 3030$, 1500, Aromatic C-H stretching, 1600-1641 $-\mathrm{CH}=\mathrm{N}, 1315-1375-\mathrm{SO}_{2-}, 3250-3330-\mathrm{NH}-$ of $-\mathrm{SO}_{2} \mathrm{NH}-, 1690 \mathrm{C}=\mathrm{O}$ of thiazolidinone, 3200,2600 -OH Phenolic (broad), 2880,2920,1400 $\mathrm{CH}_{2}, \mathrm{PMR}$ spectral Features $(\delta$ Ppm) 6.5-7.9 (multiplet, aromatic $+\mathrm{H}$ of Pyrimdine $+\mathrm{H}$ of $\left.\mathrm{SO}_{2} \mathrm{NH}\right), 6.44\left(\mathrm{H}\right.$ of $\mathrm{C}_{2} \mathrm{H}$ thiazolidinone), 3.9-4.0 (2H of $\mathrm{CH}_{2}$ for thiazolidinone), $11.34\left(\mathrm{H}\right.$ of $\left.\mathrm{SO}_{2} \mathrm{NH}\right), 2.34(3 \mathrm{H}$, singlet, $\left.\mathrm{CH}_{3}\right),{ }^{13} \mathrm{CMR}$ spectral Features $(\delta \mathrm{Ppm}) 114-131$ - Benzene, $134 \mathrm{Ar}-\mathrm{Cl}, 163-169$ - pyrimidine, 171.2$\mathrm{C}=\mathrm{O}, 33.5 \mathrm{CH}_{2}, 18-21.3 \mathrm{CH}_{3}$.

4-(2-(benzo[d][1,3]dioxol-5-yl)-4-oxothiazolidin-3-yl)-N-(4-(2,4-dichlorophenyl)-6-(6methylnaphthalen-2-yl)pyrimidin-2-yl)benzenesulfonamide $7 f$

Yield: $61 \%$, MP $188-89^{0} \mathrm{C}$, Infrared Spectral Features around $\mathrm{cm}^{-1} 3030,1500$, Aromatic C-H stretching, $1600-1641-\mathrm{CH}=\mathrm{N}, 1690 \mathrm{C}=\mathrm{O}$ of thiazolidinone, 2880,2920,1400 $\mathrm{CH}_{2}, 2950,1370-\mathrm{CH}_{3}$, 1315-1365 - $\mathrm{SO}_{2-}$, 3250-3330 -NH- of - $\mathrm{SO}_{2} \mathrm{NH}-$, PMR spectral Features $(\delta \mathrm{Ppm})$ 6.5-7.9 (multiplet, aromatic $+\mathrm{H}$ of Pyrimdine $\left.+\mathrm{H}_{\text {of }} \mathrm{SO}_{2} \mathrm{NH}\right), 6.44\left(\mathrm{H}\right.$ of $\mathrm{C}_{2} \mathrm{H}$ thiazolidinone $), 3.9-4.0\left(2 \mathrm{H}\right.$ of $\mathrm{CH}_{2}$ for thiazolidinone), 6.10 (2H for $\mathrm{CH}_{2}$ of $\left.-\mathrm{O}-\mathrm{CH}_{2}-\mathrm{O}-\right),{ }^{13} \mathrm{CMR}$ spectral Features $(\delta \mathrm{Ppm}) 114-131-$ Benzene, $134 \mathrm{Ar}-\mathrm{Cl}, 163-169$ - pyrimidine, 171.2- $\mathrm{C}=\mathrm{O}, 33.5 \mathrm{CH}_{2}, 65.6 \mathrm{CH}, 101.2 \mathrm{O}-\mathrm{CH}_{2}-\mathrm{O}-$.

N-(4-(2,4-dichlorophenyl)-6-(6-methylnaphthalen-2-yl)pyrimidin-2-yl)-4-(2-(4-hydroxy-3methoxyphenyl)-4-oxothiazolidin-3-yl)benzenesulfonamide $7 \mathrm{~g}$

Yield: $62 \%$, MP $191-92^{0} \mathrm{C}$, Infrared Spectral Features around $\mathrm{cm}^{-1} 3030$, 1500, Aromatic C-H stretching, $1600-1641-\mathrm{CH}=\mathrm{N}, 1690 \mathrm{C}=\mathrm{O}$ of thiazolidinone, 2880,2920,1400 $\mathrm{CH}_{2}, 2950,1370-\mathrm{CH}_{3}$, 1315-1365 - $\mathrm{SO}_{2}-, 3250-3330-\mathrm{NH}-$ of $-\mathrm{SO}_{2} \mathrm{NH}-, 1200$ Aryl-alkyl ether, PMR spectral Features $(\delta$ Ppm) 6.5-7.9 (multiplet, aromatic $+\mathrm{H}$ of Pyrimdine $+\mathrm{H}$ of $\left.\mathrm{SO}_{2} \mathrm{NH}\right), 6.44\left(\mathrm{H}\right.$ of $\mathrm{C}_{2} \mathrm{H}$ thiazolidinone), 3.9-4.0 (2 $\mathrm{H}$ of $\mathrm{CH}_{2}$ for thiazolidinone), $5.35(\mathrm{H}$ of $\mathrm{OH}), 3.83\left(3 \mathrm{H}\right.$ for $\left.\mathrm{OCH}_{3}\right)$, ${ }^{13} \mathrm{CMR}$ spectral Features $(\delta \mathrm{Ppm}) 114-131$ - Benzene, $134 \mathrm{Ar}-\mathrm{Cl}, 163-169$ - pyrimidine, $171.2-\mathrm{C}=\mathrm{O}$, $33.5 \mathrm{CH}_{2}, 65.6 \mathrm{CH}, 56.1 \mathrm{OCH}_{3}$.

N-(4-(2,4-dichlorophenyl)-6-(6-methylnaphthalen-2-yl)pyrimidin-2-yl)-4-(2-(3,4diethoxyphenyl)-4-oxothiazolidin-3-yl)benzenesulfonamide $7 \mathrm{~h}$

Yield: $60 \%$, MP $199-00^{0} \mathrm{C}$, Infrared Spectral Features around $\mathrm{cm}^{-1} 3030$, 1500, Aromatic C-H stretching, 1600- $1641-\mathrm{CH}=\mathrm{N}, 1690 \mathrm{C}=\mathrm{O}$ of thiazolidinone, 2880,2920,1400 $\mathrm{CH}_{2}, 2950,1370$ $\mathrm{CH}_{3}, 1315-1365-\mathrm{SO}_{2}-, 3250-3330-\mathrm{NH}-$ of $-\mathrm{SO}_{2} \mathrm{NH}-, 1200$ Aryl-alkyl ether, NMR spectral Features $\left(\delta\right.$ Ppm) 6.5-7.9 (multiplet, aromatic $+\mathrm{H}$ of Pyrimdine $+\mathrm{H}$ of $\left.\mathrm{SO}_{2} \mathrm{NH}\right), 6.44\left(\mathrm{H}\right.$ of $\mathrm{C}_{2} \mathrm{H}$ thiazolidinone), 3.9-4.0 (2H of $\mathrm{CH}_{2}$ for thiazolidinone), $5.35(\mathrm{H}$ of OH$), 1.32\left(6 \mathrm{H}\right.$ for $\left.2 \mathrm{CH}_{3}\right), 4.10$ (4H for $2 \mathrm{CH}_{2}$ ), ${ }^{13} \mathrm{CMR}$ spectral Features $(\delta \mathrm{Ppm})$ 114-131- Benzene, $134 \mathrm{Ar}-\mathrm{Cl}, 163-169$ pyrimidine, $171.2-\mathrm{C}=\mathrm{O}, 33.5 \mathrm{CH}_{2}, 65.6 \mathrm{CH}$.

\section{MICROBIAL ACTIVITY}

The compounds tested for antimicrobial activity are listed in table 4 standard drugs of compound, 5 and 6 show the size of zone of inhibition of bacterial growth procedure by test compounds for broad range of antimicrobial activity inhibiting growth of Gram-positive bacterial strains B.Subtillis and S.Aureus, and Gram-negative bacterial strains E.Coli and Ps. Aeruginosa. 
Table 4: $\quad$ Antimicrobial activity of Standards and Solvent (DMF)

\begin{tabular}{|c|l|c|c|c|c|}
\hline \multirow{2}{*}{ No. } & \multirow{2}{*}{$\begin{array}{l}\text { Name of } \\
\text { compound }\end{array}$} & \multicolumn{4}{|c|}{ Zone of inhibition (in mm) } \\
\cline { 3 - 6 } & \multicolumn{2}{|c|}{ Gram positive } & \multicolumn{2}{c|}{ Gram negative } \\
\cline { 3 - 6 } & B.Subtillis & S.Aureus & E.Coli & Ps.Aeruginosa \\
\hline 1 & DMF & 8 & 5 & 6 & 7 \\
\hline 2 & Ampicillin & 15 & 12 & 20 & 20 \\
\hline 3 & Tetracyclin & 21 & 22 & 15 & 18 \\
\hline 4 & Gentamycin & 20 & 19 & 18 & 22 \\
\hline 5 & Chloramphenicol & 21 & 23 & 17 & 24 \\
\hline
\end{tabular}

Table 5: Antimicrobial activity of 4-(arylideneamino)-N-(4-(2,4-dichlorophenyl)-6-(6-methylnaphthalen2-yl)pyrimidin-2-yl)benzenesulfonamide (6a-h)

\begin{tabular}{|c|c|c|c|c|}
\hline \multirow{2}{*}{$\begin{array}{c}\text { Compound } \\
\text { (Designation) }\end{array}$} & \multicolumn{4}{|c|}{ Zone of Inhibition (in mm) } \\
\cline { 2 - 5 } & \multicolumn{2}{|c|}{ Gram positive } & \multicolumn{2}{c|}{ Gram negative } \\
\hline B. Subtillis & S.Aureus & E.Coli & Ps.Aeruginosa \\
\hline 6a & 12 & 13 & 08 & 12 \\
\hline 6b & 10 & 12 & 10 & 10 \\
\hline 6d & 14 & 14 & 15 & 10 \\
\hline $6 \mathrm{e}$ & 10 & 10 & 08 & 09 \\
\hline $6 \mathrm{f}$ & 06 & 16 & 12 & 20 \\
\hline 6g & 13 & 11 & 10 & 14 \\
\hline $6 \mathrm{~h}$ & 21 & 19 & 14 & 17 \\
\hline
\end{tabular}

Table 6: Antimicrobial activity Synthesis of N-(4-(2,4-dichlorophenyl)-6-(6-methylnaphthalen-2yl)pyrimidin-2-yl)-4-(4-oxo-2-phenylthiazolidin-3-yl)benzenesulfonamide (7a-h).

\begin{tabular}{|c|c|c|c|c|}
\hline \multirow{2}{*}{$\begin{array}{c}\text { Compound } \\
\text { (designation) }\end{array}$} & \multicolumn{4}{|c|}{ Zone of Inhibition (in mm) } \\
\cline { 2 - 5 } & \multicolumn{2}{|c|}{ Gram positive } & \multicolumn{2}{c|}{ Gram negative } \\
\cline { 2 - 5 } & B.Subtillis & S.Aureus & E.Coli & Ps.Aeruginosa \\
\hline $7 \mathrm{a}$ & 09 & 12 & 11 & 17 \\
\hline $7 \mathrm{~b}$ & 14 & 09 & 13 & 09 \\
\hline $7 \mathrm{c}$ & 13 & 12 & 08 & 08 \\
\hline $7 \mathrm{~d}$ & 14 & 10 & 20 & 13 \\
\hline $7 \mathrm{e}$ & 18 & 14 & 14 & 20 \\
\hline $7 \mathrm{f}$ & 22 & 20 & 18 & 13 \\
\hline $7 \mathrm{~g}$ & 14 & 16 & 15 & 21 \\
\hline $7 \mathrm{~h}$ & 15 & 15 & 19 & \\
\hline
\end{tabular}

\section{CONCLUSIONS}

In conclusion, a new series of compound $6(\mathrm{a}-\mathrm{h})$ and $7(\mathrm{a}-\mathrm{h})$ were synthesized, compounds screened for their spectral study and biological study. The investigation of antimicrobial activities data revealed that the compounds $(7 \mathrm{~d}),(7 \mathrm{e}),(7 \mathrm{f}),(7 \mathrm{~g})$ and $(7 \mathrm{~h})$, displayed excellent activity, the compounds(7a), (7b) and (7e) showed moderate activity and rested compounds showed less activity compared with standard drugs.

\section{ACKNOWLEDGEMENTS}

We heartily thankful to Department of Chemistry for providing elemental analysis and also thankful to Head, CDRI, Lucknow for providing spectral data for the compounds. 


\section{References:}

[1] C. Frances Brown, 4-Thiazolidinones. Chemical Reviews 19661 (5) 463-521.

[2] A.K. Jain, A. Vaidya, V. Ravichandran, S.K. Kashaw, Recent developments and biological activities of thiazolidinone derivatives: A review. Bioorganic \& Medicinal Chemistry, 20 (2012) $113378-3395$.

[3] S.S. Syed, T.R. Radhakrishnan. Studies on biologically active heterocycles. Synthesis and antibacterial activity of some 2,5-disubstituted-1,3,4-oxadiazole, 1,3,4- thiadiazole,1,2,4triazole and 4-thiazolidinones. Indian Journal of Heterocyclic Chemistry, 5 (1995) 133-138.

[4] B.S. Vashi, D.S. Mehta, V.H. Shah, Synthesis and biological activity of 4-thiazolidinones, 2azetidinones, 4-imidazolinone derivatives having thymol moiety. Indian Journal of Chemistry, 34B (1995) 802-808.

[5] A.H. Bhatt, K.A. Parikh, A.R. Parikh, Synthesis of some thiazolidinones and 5oxoimidazolines as biologically potent agents. Indian Journal of Chemistry, 38(B) (1999) 628631.

[6] V.J. Sattigeri, A. Soni, S. Singhal, S. Khan, M. Pandya, Synthesis and antimicrobial activity of novel thiazolidinones. Arkivoc, 2 (2005) 46-59.

[7] M. Amir, A. Faizul. Synthesis and biological evaluation of some 4-thiazolidinones. Indian Journal of Heterocyclic Chemistry, 14 (2004) 119-122.

[8] R.P. Singh, D.V. Singh, C.R. Singh, S.P. Tripathi, S. Singh, Synthesis and antifungal activity of 2-azetidinones, 4-thiazolidinones and 5-imidazolidinones incorporating benzthiazole moiety. Journal of Chemical and Pharmaceutical Research, 4, 4 (2012) 2055-2060.

[9] A.V. Dobaria, J.R. Patel, H.H. Parekh, Thiazolidinones bearing chloro quinolone nucleus as potential antimicrobial agents. Indian Journal of heterocyclic chemistry. 11 (2001) 115.

[10] A.K. Solonkee, I. Thakor, J. Patel, S. Lad, Synthesis and Biological assay of some new 2,3Diaryl-5-methyl-4-thiazolidones. Oriental J. chemistry, 201 (2004) 127.

[11]P. Mishra, K.P. Namdeo, S.K. Jain, S. Jain, synthesis and Antimicrobial Activity of 4thiazolidinone. Asian J. chem, 111 (1991) 55.

[12]N.J. Gaikward, P. Gautam, Mannich reaction products of 5-Benzylidine-2-Thioxo-4thiazolidinone as hypoglycemic agents, Indian J. Heterocyclic chem., 2002;12:181-182.

[13]D.J. Kaneria, N.J. Data, R.C. Khunt, N.H. Paresh, Synthesis and pharmacological activity of some new 4-thiazolidinone derivatives. Indian J. Heterocyclic chem, 12 (2003) 277.

[14]N. Rama Rao, B. Ramu, C. Gopinath, Synthesis and charectarisation of some biological thiazolidene. Asian Journal of chemistry, 1 (2004) 531.

[15]D.S. Nair, A.C. Shah, Synthesis of 5-thiazolidinone derivatives of (R) and (S) -2aminobutanols. Indian Journal of heterocyclic chemistry, 16 (2007) 231.

[16]C.D. Diulatbad, G.G. Bhat, Synthesis and antimicrobial studies of 4-Thiazolidinone derivatives from 2-Bromohexanoic acid (part II). Indian Journal of heterocyclic chemistry, 9 (1999) 157.

[17] Giri, M.H. Khan, Synthesis and antifungal activity of some 3-[5'-aryl-3'-Mercapto- 1',2',4'triazol-4-yl]-4-Thiazolidinones. Asian J.chem,4 4 (1992) 812. 